Chirurg 2019 $90: 241$

https://doi.org/10.1007/s00104-019-0806-7

Online publiziert: 7. Februar 2019

(c) Springer Medizin Verlag GmbH, ein Teil von Springer Nature 2019

CrossMark

\author{
Aladdin Ali Deeb - Michael Ardelt - Utz Settmacher \\ Klinik für Allgemein-, Viszeral- und Gefäßchirurgie, Jena, Deutschland
}

\title{
Auswirkung von Komplikationen nach Leberresektionen
}

tengruppen. Die statistische Signifikanz wurde als $p<0,05$ definiert.

Chacon E, Vilchez V, Eman P et al (2018) Effect of critical care complications on perioperative mortality and hospital length of stay after hepatectomy: a multicenter analysis of 21,443. patients. Am J Surg. https://doi.org/ 10.1016/j.amjsurg.2018.11.016

Hintergrund. Von 2000 bis 2010 wurden zunehmend Leberteilresektionen in den USA durchgeführt. Die perioperative Mortalität hat sich in den letzten vier Jahrzehnten von $17 \%$ auf unter $5 \%$ deutlich verringert. Unter anderem hat das perioperative Management dazu beigetragen, Morbidität und Mortalität zu reduzieren. Ziel dieser Studie ist es, perioperative Risikofaktoren zu identifizieren, die an der Entwicklung schwerer postoperativer Komplikationen beteiligt sind - hier als „critical care-complications" (CCC) definiert - und deren Auswirkungen auf die Krankenhausaufenthaltsdauer und die perioperative Mortalität nach Leberresektion zu bewerten.

Methodik. Alle Leberesektionspatienten $(n=21.443)$ in der NSQIP-Datenbank (nationales chirurgisches Qualitätsverbesserungsprogramm des American College of Surgeons) von 2012 bis 2016 wurden analysiert. Der primäre Endpunkt der Studie war die Ausbildung einer der folgenden intensivmedizinischen Komplikationen (CCC): verlängerte Beatmung ( $>48 \mathrm{~h})$, Sepsis/septischer Schock, Nierenversagen, Herzstillstand/ Myokardinfarkt oder Lungenembolie. Die intra- und perioperativen Charakteristika der CCC-Gruppe wurden mit einer Kontrollgruppe verglichen, deren Patienten keine CCC entwickelten. Zusätzlich erfolgte eine Analyse der perioperativen Mortalität und Krankenhausaufenthaltsdauer der beiden Patien-
Die Gesamtmortalität betrug $n=342$ (1,6\%). In der CCC-Gruppe betrug diese $n=299$ (12,7\%).

Patienten mit mindestens einem CCC hatten in der Durchführung einer Propensity-Score-Analyse ein 26-fach erhöhtes Mortalitätsrisiko $(p<0,0001)$ und eine verlängerte Krankenhausaufenthaltsdauer von 10,5 Tagen über dem Mittelwert $(p<0,0001)$ im Vergleich zur Kontrollgruppe.

Diskussion und Fazit. Diese Studie analysiert Prädiktoren für schwerwiegende Komplikationen und deren Einfluss auf dieperioperative Mortalitätund dieKrankenhausaufenthaltsdauer nach Leberresektionen. Wahrscheinlich wird die präoperative Optimierung dieser Prädiktoren vor elektiven Leberresektionen einen positiven Einfluss aufdie Prognosehaben.

Um den Einfluss der präoperativen Ernährungsoptimierung sowie der Physiotherapie auf die Prognose nach Leberresektion zu beurteilen, bedarf es einer prospektiven Studie. Die Studienergebnisse deuten darauf hin, dass die Diabetes- und Blutdruckkontrolle sowie die kardiopulmonale Optimierung eine Rolle bei der Verringerung schwerer Komplikationen bei Patienten mit Leberresektion spielen könnten.

\section{Korrespondenzadresse}

PD Dr. med. habil. Dr. med. univ. Michael Ardelt

Klinik für Allgemein-, Viszeral- und Gefäßchirurgie

Am Klinikum 1, 07747 Jena, Deutschland Michael.Ardelt@med.uni-jena.de

Interessenkonflikt. A.A. Deeb, M. Ardelt und U. Settmacher geben an, dass kein Interessenkonflikt besteht. 